\title{
Los Departamentos Municipales de Educación en Galicia en la legislatura 2007-2011: un estudio de casos
}

\section{Os Departamentos Municipais de Educação na Galícia na legislatura 2007-2011: um estudo de casos}

\section{Municipal Departments of Education in Galicia in the 2007-2011 term: a case study}

\author{
Francisco Mareque León ${ }^{1}$ \\ María Belén Caballo Villar ${ }^{1}$ \\ Jesús Rodríguez Rodríguez ${ }^{1}$
}

\begin{abstract}
RESUMEN
En un contexto europeo en el que las políticas educativas evolucionan hacia la cogestión y la colaboración multinivel, el presente estudio analiza la situación de los Departamentos Municipales de Educación en Galicia (España), considerándolos una pieza clave para el desarrollo de proyectos educativos de territorio que vertebren el futuro de las políticas educativas locales. El estudio, que combina técnicas cuantitativas y cualitativas de recogida de datos, analiza el trabajo realizado en los trece departamentos identificados en Galicia en el año 2007-2011. Los resultados demuestran las diferencias existentes entre la oferta educativa al alcance de los ciudadanos residentes en los distintos municipios, lo que lleva a plantearse la necesidad de trabajar de un modo colaborativo y organizado en pro de una mayor optimización de recursos e igualdad de oportunidades que garanticen el derecho a la educación.
\end{abstract}

Palabras-clave: política; educación; municipio; cogestión; colaboración.

DOI: $10.1590 / 0104-4060.47174$

1 Universidade de Santiago de Compostela. Facultad de Ciencias de la Educación. Santiago de Compostela, A Coruña, España. Rúa Xosé María Suárez Núñez, s/nº Campus Vida, 15782. E-mails: fmareque@edu.xunta.es; belen.caballo@usc.es e jesus.rodriguez.rodriguez@usc.es 


\title{
RESUMO
}

Num contexto europeu, em que as políticas educacionais evoluem para a cogestão e a colaboração multinível, o presente estudo analisa a situação dos Departamentos Municipais de Educação na Galícia (Espanha), considerando-os uma peça-chave para o desenvolvimento de projetos educacionais de território que alicerçam o futuro das políticas educativas locais. $\mathrm{O}$ estudo, que combina técnicas quantitativas e qualitativas de coleta de dados, analisa o trabalho efetuado nos treze departamentos identificados na Galicía na legislatura 2007-2011. Os resultados mostram as diferenças existentes na oferta educativa ao alcance dos cidadãos residentes nos diversos municípios, o que leva a considerar a necessidade de trabalhar de forma colaborativa e organizada em prol de uma maior otimização dos recursos e igualdade de oportunidades que garantam o direito à educação.

Palavras-chave: política; educação; município; cogestão; colaboração.

\begin{abstract}
In a European context where educative policies evolve towards codetermination and horizontal and vertical collaboration, this study analyzes the status of the Municipal Departments of Education in Galicia (Spain), considering them a key piece for the development of fundamental educational territorial projects for the future of local educational policies. The study combines quantitative and qualitative data collecting devices, analyzing the work done in thirteen departments identified in Galicia in the 20072011 term. The results demonstrate the differences between educational opportunities available to citizens in different municipalities, which makes us ask ourselves about the need to work in a collaborative and organized way for greater optimization of resources and equal opportunities among citizens that ensure the right to education.
\end{abstract}

Keywords: politics; education; municipality; co-determination; collaboration.

\section{Introducción}

En las últimas décadas parece constatarse en la Unión Europea un proceso tendente a impulsar una activa colaboración entre Administraciones públicas, entidades sociales e iniciativas personales en el desarrollo de las políticas educativas. Autores reconocidos en este campo de la literatura, como Colino y Del Pino (2008); Díaz-Gibson et al. (2015); Montero y Caballo (2011), entre 
otros, así lo reflejan en sus obras. El origen de estas tendencias se encuentra en los procesos de descentralización que potencian la cogestión y la participación social, optimizando recursos y consiguiendo mejores resultados en un escenario que obliga a someter a reflexión los modelos pretéritos. Y es en este proceso donde los Departamentos Municipales de Educación (DME) juegan un papel esencial como coordinadores de los agentes implicados en el campo educativo de su ámbito territorial.

Fruto de esta situación nace un estudio de casos sobre las políticas educativas y los DME en Galicia, que asume como objetivo general analizar la situación de los mismos en la legislatura 2007-2011 para esbozar líneas de desarrollo que orienten su trabajo futuro en el marco de las políticas educativas locales en esta comunidad autónoma.

\section{Fundamentación}

La relación existente entre la Educación y la Política, en una sociedad concreta, condiciona significativamente la actividad de todos los agentes educativos de la misma. Para Subirats (2003), una vez superado el concepto educativo basado exclusivamente en el sistema escolar, y hecha la constatación de que la educación afecta a todos los ámbitos sociales, procede la reflexión para definir cuál tiene que ser la política educativa y cómo aplicarla.

Y en este sentido, el trabajo de los DME tiene mucho que ver con el modelo conceptual de relación educación-territorio por el que se opte en cada contexto; encontrándose estos nexos fuertemente condicionados por las voluntades y actitudes políticas. Es por ello que los continuos cambios sociales obligan a una reconceptualización, retroalimentación y adecuación de las distintas políticas a las nuevas demandas y necesidades, en la línea de lo señalado por autores como Subirats (2007) o Galais, Navarro Yáñez y Fontcubierta (2013). En este proceso existen diversos factores determinantes para el futuro de las políticas educativas, entre ellos, la participación social como verdadero eje de la democratización de la vida pública; y la descentralización de competencias.

En lo referente a la descentralización, el proceso de transferencia de competencias educativas a las Comunidades Autónomas vivido en las últimas décadas, hace necesario estar atentos a no caer en un policentrismo autonómico. En contextos democráticos, las tendencias descentralizadoras se originan y se mantienen prioritariamente desde presupuestos de participación de los sectores implicados, tal y como indica Espejo (2001), de ahí que las iniciativas no 
deban subyugarse al monopolio de ninguna administración. Por el contrario, un proceso de corresponsabilidad política entre administraciones, donde la ciudadanía pueda participar activamente resulta, a nuestro entender, la fórmula más adecuada. Como indica Bonal (2007, p. 52), “[...] es imprescindible una verdadera voluntad de cogestión mediante un proceso de descentralización y no de simple desconcentración o delegación de responsabilidades".

En esta línea, si analizamos las políticas educativas de territorios y países de la Unión Europea, podremos comprobar cómo - en líneas generales - parece existir una evolución hacia sistemas municipalistas de mayor descentralización, donde los consecutivos informes Programme for International Student Assessment (PISA) apuntan una correlación significativa con los mejores resultados.

Por lo tanto, en líneas generales, y a la luz de los diversos estudios realizados en este campo, consideramos que a medio plazo, si existen las voluntades políticas necesarias, debería darse en Galicia un acercamiento progresivo a procesos de segunda descentralización, cogestión y potenciación de la participación ciudadana en las políticas educativas, cobrando especial relevancia el papel de los ayuntamientos, y, en consecuencia, de los DME (en línea con lo señalado en el Pacto Local firmado en Galicia el 20 de Enero de 2006). Sus competencias, cuyas aportaciones provienen de la Ley Orgánica de Educación - LOE (2006), deberán evolucionar con la firma de nuevos acuerdos entre administraciones y colectivos sociales, posibilitando la realización de Proyectos Educativos Territoriales compartidos.

Los DME nacen en nuestro país fruto de una demanda social basada en múltiples necesidades, entre las que destaca la urgencia de armonizar la actividad desarrollada desde los ayuntamientos democráticos que se constituyen en 1979 y las acuciantes carencias sociales de la España de la transición. Ante esta situación, y previo a aprobase la Ley Reguladora de Bases de Régimen Local, 1985, la acción que se desarrollará desde los ayuntamientos va a consistir, según las actas de las I Jornadas gallegas sobre Ayuntamientos y Educación (VVAA, 1985), en una serie de actividades aisladas, individuales e inconexas, que intentan dar solución a todas aquellas problemáticas hasta entonces desatendidas.

Los Servicios Municipales surgen, entonces, según Candedo y otros (1990, p. 257), como unidades especializadas, asumiendo en el caso de la educación competencias de gestión general y específica, planificación y dinamización educativa o participación ciudadana. En ese momento los DME existentes en España adquieren, en general, una estructura con presupuesto específico y funciones diferenciadas, destacando tres áreas que se concretan en las unidades de obras; dinamización y planificación educativa; y gestión y coordinación.

Los inicios de los DME no fueron sencillos, pero conformaron las bases de las políticas municipales actuales. En este sentido cabe destacar la labor de- 
sarrollada en estos años por la Federación Española de Municipios y Provincias (FEMP) en pro de las competencias, funciones y condiciones de trabajo de los municipios en materia de educación.

Teniendo presente este proceso, podemos afirmar que la situación de las competencias educativas municipales, y en consecuencia de los DME, vivió una significativa evolución en las últimas décadas, aun constatando la existencia de limitaciones y diferencias en las distintas Comunidades Autónomas. No obstante, coincidimos con Caballo (2009), cuando afirma que la situación educativa de nuestros ayuntamientos continúa caracterizada por una escasez de competencias de titularidad municipal que, en su conjunto, no llegan a satisfacer el principio de autonomía local. La nueva Ley 27/2013, de 27 de diciembre, de racionalización y sostenibilidad de la Administración local, comentada por Quintana y Casares (2014), se caracteriza por el estancamiento en el proceso descentralizador realizado por parte de la Administración autonómica en este campo; también por infrautilizar las posibilidades existentes para activarla. Solo una verdadera voluntad de la clase política podría desbloquear esta tendencia.

En un análisis más general de la situación de la educación destacan las aportaciones del profesor Subirats, que insite en la necesidad de tener presente el cambio de época que vivimos a la hora de analizar la realidad y formular las medidas que se deben tomar en el campo educativo local. Según este autor, para entender la situación de estos departamentos debemos tener en cuenta las transformaciones que están afectando a la esfera productiva, a la estructura social y familiar, así como a las políticas públicas tradicionales. En definitiva, y en este nuevo marco, "una buena educación es el conjunto de una buena relación y de un buen trabajo en equipo entre la familia, la localidad, el barrio y la escuela", tal y como indicaba en su intervención Subirats en seminario sobre Educación y Administración local: Propuestas de acción social y comunitaria organizado por la Universidade Internacional Menéndez Pelayo y celebrado en A Coruña, del 14 al 18 de Septiembre de 2009.

En todo momento lana escuela debe participar en conjunto con los centros educativos. Y en esta línea, compartimos con el citado autor la necesidad de trabajar en red; una red en la que la escuela desarrolle su labor con el resto de sectores sociales implicados en el proceso educativo, y en el que los servicios municipales y el territorio cobren un valor significativo. Admitiendo como válida esta premisa, se reafirma la importancia de estos departamentos en nuestros ayuntamientos: si la evolución de la sociedad nos lleva a trabajar los procesos educativos en red, no se podría entender el futuro de las políticas educativas en nuestro país alejadas del papel que los ayuntamientos pueden y deben asumir en tal proceso. 


\section{Metodología}

Para la consecución del objetivo general, centrado en el análisis de los DME en Galicia en el período 2007-2011 y el diseño de unas orientaciones para su desarrollo, formulamos una serie de objetivos específicos que se concretan en analizar sus recursos humanos, materiales y económicos; estudiar su actividad en los ámbitos de gestión, planificación y dinamización de actividades; profundizar en la perspectiva de los responsables políticos y técnicos sobre la situación presente y futura de estos departamentos; y avanzar sugerencias y estrategias que faciliten, en clave prospectiva, su trabajo y la evolución de las correspondientes políticas educativas.

La delimitación de la muestra resultó una tarea compleja, pues no existía un listado actualizado de los municipios gallegos que especificase si contaban con departamento de educación; por otra parte, muchos ayuntamientos derivan las competencias educativas a otras secciones municipales, mayoritariamente a las concejalías o departamentos de cultura, juventud o deportes. No obstante, y puesto que nuestro objetivo se ciñe exclusivamente a los DME, tras revisar los organizagramas de los 315 ayuntamientos gallegos, se excluyeron aquellos que no explicitaban la categoría "educación" en el mismo, así como los que no contaban por lo menos con un técnico (aspecto confirmado telefonicamente).

Con el fin de lograr un listado completo y actualizado, realizamos una búsqueda en las páginas web de cada ayuntamiento (en muchas ocasiones sin datos al respecto), en diferentes instituciones y organismos (Xunta de Galicia, Diputaciones Provinciales, Federación Gallega de Municipios y Provincias FEGAMP) y a través de profesorado universitario especialista en el tema.

Finalmente, y tras contrastar la información aportada por las distintas fuentes, identificamos como DME en Galicia en el año 2007, con por lo menos un técnico municipal adscrito, los siguientes: A Coruña, Ames, Betanzos, Boqueixón, Lugo, Narón, Oleiros, Ourense, Pontevedra, Santiago de Compostela, Sanxenxo, Vigo y Vilagarcía de Arousa.

Ante esta realidad, se optó por una metodología de investigación de estudio de casos, pues representa, en determinada medida, la realidad (MENDOZA, 2006, p. 39), y por lo tanto supone una cierta complejidad. Dentro de la diversidad de tipologías, este estudio se encuadra en un modelo evaluativo, según la clasificación de Guba y Lincoln (1981, p. 374); por otra parte, y siguiendo la taxonomía de Bogdan y Biklen (1982), estamos ante un estudio múltiple, ya que empleamos varios casos únicos al mismo tiempo para analizar la realidad que queremos investigar. A la par se define como global o colectivo, pues con- 
sideramos de una manera conjunta nuestra realidad de análisis, en este caso los DME en Galicia.

Por lo tanto, y en coherencia con las diferentes fases descritas por los autores anteriormente citados, estructuramos el análisis de estos departamentos en las siguientes etapas: exploración del estudio; descripción y explicación; evaluación de los resultados; conclusiones; prospectiva y propuestas de mejora.

Habida cuenta de los objetivos específicos definidos, los instrumentos (cuestionario y entrevista) se estructuraron en cuatro bloques de contenido: el primero hace referencia a los recursos económicos, humanos y materiales de los departamentos; el segundo se centra en las actividades de gestión, planificación y dinamización de las mismas; el tercero alude a las políticas educativas que enmarcan su funcionamiento; y por último, se procuró esbozar líneas de avance para el trabajo a medio plazo de estos departamentos.

En nuestro estudio optamos, pues, por dos instrumentos fundamentales: el cuestionario y la entrevista. Con el cuestionario intentamos recopilar toda la información sobre nuestros dos primeros objetivos específicos, los recursos y las actividades realizadas. Fue construído para ser aplicado a todos los directores técnicos de los departamentos de educación que componen los casos de investigación, y en su apartado final se indaga sobre sus opiniones en torno a las perspectivas de futuro.

Complementariamente, se realizó una entrevista semiestruturada a los responsables políticos de educación, con la finalidad de que nos ofrecieran su visión sobre la situación de los DME en Galicia. Esta técnica permitió profundizar en los dos últimos aspectos señalados anteriormente: la influencia política sobre los departamentos de educación y sus perspectivas de futuro. De igual modo posibilitó el contraste de opiniones entre los responsables políticos y técnicos sobre la situación de la legislatura 2007-2011, funcionamiento y prospectiva.

Para la aplicación de los dos instrumentos se procedió, en el caso de las entrevistas, a concertar una cita vía telefónica con la suficiente antelación para desplazarse a los municipios en las fechas acordadas; por su parte, los cuestionarios fueron enviados por correo electrónico a los/las jefes/as o directores/as de los departamentos municipales de educación, acompañados de la correspondiente carta explicativa de presentación.

\section{Interpretación y discusión de los resultados}

Presentamos los resultados del estudio agrupados en cinco apartados: datos de los municipios con departamento de educación; perfil de los respon- 
sables políticos y técnicos; recursos; actividad desarrollada; y opinión de los responsables de los departamentos sobre la situación y futuro de los mismos.

En lo referente a los datos de ayuntamientos con DME, identificamos en Galicia un total de trece que los incorporan en su organigrama, contando, además, y por lo menos, con un técnico adscrito a mayores del responsable político correspondiente. Así, apenas un 4\% de los ayuntamientos gallegos disponen de un departamento específico de educación. De los trece municipios, seis se corresponden con grandes urbes gallegas, y siete son de entidad media o pequeña, caracterizados por una notable implicación desde el ámbito político. Se comprueba, pues, que la relación entre la dimensión del municipio y el modo de abordar sus competencias es manifiesta.

Otro aspecto a subrayar es la situación geográfica de estos ayuntamientos. Once se encuadran en el conocido como corredor atlántico, en la Galicia costera, más favorecida que la interior tanto en vías de comunicación como en desarrollo económico o en apoyo histórico de las instituciones, lo que lamentablemente dio lugar a una Galicia de dos velocidades; una dualidad también palpable en el campo de las políticas educativas municipales. En la zona interior, tan sólo las dos capitales de provincia cuentan con un departamento específico centrado en el campo educativo.

En el desarrollo de la investigación pudimos acercarnos a las directrices y características fundamentales respeto a la filosofía de la Red Internacional de Ciudades Educadoras. En el estudio corroboramos la existencia de ocho ciudades miembro en Galicia, todas ellas situadas en este corredor atlántico, coincidiendo en seis ocasiones con las principales ciudades gallegas.

Otro de los aspectos que destacamos es la implicación política. En el momento de la realización del trabajo de campo, año 2007, gobernaba casi en un $80 \%$ de los ayuntamientos con DME un partido político de carácter progresista; siendo esta misma tendencia la que gobernaba en la mayoría de los ayuntamientos en el momento de la creación de estos departamentos. Por lo tanto, podemos afirmar que la génisis y evolución de un DME vino determinada por la concienciación e implicación de los responsables políticos respecto de la importancia de los procesos educativos locales.

En segundo lugar, en lo referente al perfil de sus responsables técnicos y políticos, encontramos importantes analogías entre ambos: persona de mediana edad, con preparación universitaria y dedicación exclusiva al cargo. Los técnicos son mayoritariamente hombres (65\%), mientras que en el caso de los/las ediles predomina la figura femenina $(62,5 \%)$. Los responsables técnicos aseguran estar especialmente condicionados por los responsables políticos del área, mientras éstos afirman mayoritariamente contar con el apoyo del grupo de gobierno para 
el desempeño de su actividad. La relación entre ambos, en términos generales, puede considerarse buena.

En tercer lugar, cuando hacemos referencia a los recursos, una vez más se hacen palpables las grandes diferencias en función de las dimensiones municipales. Los recursos de cada ayuntamiento son una de las principales limitaciones para el desarrollo de las competencias en materia de educación. Si por un lado encontramos ayuntamientos que destinan importantísimas cantidades de fondos económicos, humanos y materiales al ámbito educativo; por otro apreciamos municipios literalmente ahogados por las competencias que, como el mantenimiento de los centros públicos de educación infantil y primaria, les son asignadas sin adjudicarles los recursos suficientes para su cumplimiento, lo que en el caso de los municipios de menor entidad limita significativamente la posibilidad de realizar ningún otro tipo de actividad; en la línea de lo ya indicado hace más de dos décadas por Costa (1985, p. 121), y más recientemente por Rodríguez-Abella (2003, p. 54). En este sentido, constatamos una media destinada al ámbito educativo en el presupuesto municipal del 8,6\% entre los municipios analizados, con variaciones entre el $2 \%$ y $34 \%$ en los casos extremos. Respecto a la variación en el incremento del presupuesto para esta partida en los años anteriores, las diferencias se sitúan entre el $1 \%$ y el $18 \%$, encontrando la media en el 3\% positivo anual. Analizados los datos del estudio, entendemos que este fenómeno podría deberse, además de a una disponibilidad presupuestaria mayor, a que es en estos ayuntamientos donde las necesidades básicas de la población frecuentemente demandadas (saneamiento integral, infraestructuras viarias...) están mejor cubiertas, lo que posibilita destinar más fondos a otras áreas, en la línea de lo indicado por Méndez Romeu (2002).

En paralelo a los recursos económicos, todos los departamentos demandan una ampliación de personal, especialmente técnicos especializados, y, en menor medida, mayor dotación de recursos materiales. En cuanto a estos últimos, de nuevo se constata la diferencia entre los ayuntamientos de mayor población, que en líneas generales están satisfechos, con los de menor entidad, que solicitan un incremento de dotación.

En cuarto lugar, respecto a la actividad desarrollada, podemos confirmar que todos ellos cumplen con las funciones y competencias que legalmente les son asignadas en materia educativa, tal y como podemos observar porcentualmente en la tabla siguiente. No obstante, una vez más las diferencias se hacen patentes dependiendo de las dimensiones poblacionales del municipio que analicemos. 
TABLA 1 - PORCENTAJE DE DESARROLLO DE COMPETENCIAS EDUCATIVAS

\begin{tabular}{|c|c|c|c|c|c|}
\hline & $\begin{array}{l}\text { Muy } \\
\text { alto }\end{array}$ & Alto & Medio & Bajo & $\begin{array}{l}\text { Muy } \\
\text { bajo }\end{array}$ \\
\hline $\begin{array}{l}\text { Vehicular la relación entre la programación de } \\
\text { los centros y su ámbito socioeconómico en el que } \\
\text { desarrollan su labor }\end{array}$ & 18,2 & 45,5 & 18,2 & - & 18,2 \\
\hline $\begin{array}{l}\text { Colaborar con los centros en el impulso de } \\
\text { actividades complementarias y extraescolares }\end{array}$ & 33,3 & 41,7 & 25 & - & - \\
\hline $\begin{array}{l}\text { Uso de centros fuera del horario lectivo, para } \\
\text { otras actividades }\end{array}$ & 8,3 & 58,3 & 8,3 & 16,7 & 8,3 \\
\hline Cooperar en los programas de garantía social & - & - & - & 30 & 70 \\
\hline $\begin{array}{l}\text { Colaborar con las administracións educativas en } \\
\text { la formación del profesorado }\end{array}$ & - & 8,3 & - & 58,3 & 33,3 \\
\hline Colaborar en la educación infantil & 33,3 & 25 & 16,7 & 16,7 & 8,3 \\
\hline Colaborar en la educación primaria & 25 & 25 & 33,3 & 16,7 & - \\
\hline Colaborar en la educación secundaria & 8,3 & 8,3 & 66,7 & 16,7 & - \\
\hline Colaborar en la educación xuvenil & - & 9,1 & 63,6 & 9,1 & 18,2 \\
\hline Colaborar en la educación de adultos & 8,3 & 25 & 8,3 & 33,3 & 25 \\
\hline $\begin{array}{l}\text { Colaborar con las enseñanzas de régimen especial } \\
\text { como conservatorios de música y danza }\end{array}$ & 36,4 & 27,3 & 9,1 & 18,2 & 9,1 \\
\hline
\end{tabular}

FUENTE: Los autores (2016). Elaboración a partir de cuestionario a técnicos municipais.

En el marco de la actividad educativa, tal y como afirmamos con anterioridad, existe una competencia asignada a los municipios que condiciona significativamente el funcionamiento de los DME, acaparando la inmensa mayoría de los recursos disponibles, en especial en los municipios más humildes. Estamos haciendo referencia al mantenimiento y conservación de los centros públicos de educación infantil y primaria.

No obstante, es preciso resaltar que todos los ayuntamientos de nuestra Comunidad Autónoma cumplen esta función con independencia del grupo político que gobierne, o de la entidad en dimensión de población o territorio del ayuntamiento. En este sentido, consideramos necesario hacer referencia al acuerdo firmado en 2006 por la FEGAMP (Federación Gallega de Municipios y Provincias) y la Xunta de Galicia (Consellería de Educación), con el cual se consiguió la asunción de ciertos gastos de mantenimiento de los centros de enseñanza primaria con la creación de una comisión mixta paritaria encargada 
de elaborar propuestas que permitan que la Xunta de Galicia palíe ciertos gastos económicos que soportan los ayuntamientos.

Al margen de esta competencia específica, los DME en Galicia centran su actividad en ayudar a conciliar la vida laboral y familiar apoyando la educación infantil, la potenciación de actividades extraescolares, música, danza y el uso de los centros escolares fuera del horario lectivo. También destaca el volumen de programas y actividades dirigidas a dar a conocer el propio municipio. Se constata una mayor proliferación de actividades y programas en aquellos más privilegiados económicamente, produciendo de por sí una situación de desigualdad manifiesta entre ciudadanos y ciudadanas en función de su municipio de residencia.

Las actividades suelen realizarse mayoritariamente en dependencias municipales, centros educativos y en espacios exteriores del propio municipio. Se observa un mayor volumen de actividades y programas centrados en la educación reglada, lo cual, a nuestro entender, merece una reflexión, pues resulta imprescindible entender la educación como un proceso global más allá del contexto escolar, en la línea del señalado hace décadas por Dewey, Alfieri o la filosofía de la Ciudad Educadora y de los Proyectos Educativos de Ciudad; y en este sentido los DME pueden jugar un papel clave.

Por otro lado, mayoritariamente $(62,5 \%)$, podemos hablar de un modelo de gestión mixto, que combina actividades desarrolladas por el propio departamento y las subcontratadas a agentes externos. En un análisis más detallado apreciamos que son los municipios de menores recursos los que optan en mayor medida por la gestión directa ( $37,5 \%$ de los casos), incrementándose el grado de externalización en aquellos de mayores posibilidades y volumen de trabajo. En este sentido debemos tener presente que otro factor que incide en la elección del modelo de gestión es la ideología y la visión política que de la administración tenga el grupo de gobierno correspondiente. De ahí que los cambios de gobierno den lugar, en muchas ocasiones, a modificaciones en dicho modelo.

En todo el estudio se aprecia la intensa relación de estos departamentos con otros de su mismo ayuntamiento, no siendo así con otros niveles de la Administración con los que apenas realizan colaboraciones puntuales. La colaboración con entidades a la hora de realizar programas o actividades es baja $(46,2 \%)$ o muy baja $(38,5 \%)$, denotando cierto aislamiento de estos departamentos en relación al tejido social. Consideramos pues, que la colaboración interinstitucional podría enriquecer y complementar la oferta existente. Sin duda, este apartado será en el futuro próximo uno de los elementos que deba marcar la evolución de los DME en Galicia; en la línea de la denominada cogestión, aplicada ya en otros territorios y definida por una estrecha coordinación entre Administraciones públicas. 
Cuando nos referimos al modo de trabajo, planificación y gestión apreciamos que, mayoritariamente, en un $46,2 \%$ de los municipios, se realizan reuniones para planificar el trabajo de modo semanal. Resulta llamativo que en el $23 \%$ de los casos se afirma no llevar a cabo estas reuniones, realidad que atribuimos a departamentos con un volumen de personal y dimensiones relativamente pequeños, en los cuales el contacto es directo y continuo entre sus miembros.

No se realizan estudios previos a la toma de decisiones en un $63 \%$ de los casos, lo cual implica que en muchas ocasiones se pueda actuar basándose en criterios personales del responsable técnico o político. Un 84,6\% de los técnicos considera necesaria la realización de mapas escolares en sus municipios, no obstante, en el $30,8 \%$ de los casos no existen.

Del conjunto de actividades, en las fechas estudiadas el $72 \%$ fueron gratuitas para sus usuarios, el 19\% subvencionadas y el 7,5\% de pago. Con estos resultados es fácil comprender por qué las cuotas de usuarios no son una fuente significativa de ingresos. De igual modo se infiere un modelo de gestión pública dirigida a una oferta de actividades extensa y gratuita para la población, lo que inicialmente supone un estímulo para el incremento de la participación.

$\mathrm{Y}$ en quinto lugar, respeto a la situación y futuro de los DME, se pueden extraer varias conclusiones:

Cuando preguntamos a los responsables políticos y técnicos de estos departamentos sobre la situación y futuro de los mismos, afirman que existen tres problemas fundamentales en el ámbito educativo local: 1) La ambigüedad del marco legislativo y de las competencias aplicables; 2) la carencia de recursos para el correcto desarrollo de las competencias asignadas; y 3 ) en consecuencia, la inexistencia o inestabilidad de los DME en la mayoría de los ayuntamientos gallegos.

En esta línea, estos responsables abogan por un nuevo debate para la clarificación, determinación y reconocimiento de las competencias locales en materia de educación; lo cual necesariamente debe ir acompañado de la dotación presupuestaria correspondiente. En algunos casos, esencialmente los ayuntamientos con mayores medios se plantean la posibilidad de una mayor descentralización de competencias, alcanzando la Administración local el rango de Administración educativa, tal y como ocurre en otros sistemas europeos. Desafortunadamente, ni la Ley Orgánica 8/2013, para la Mejora de la Calidad Educativa (LOMCE), ni la Ley 27/2013 de Racionalización y Sostenibilidad de la Administración Local avanzan en esta dirección.

Por último, todos los responsables, políticos y técnicos, defienden un modelo de trabajo en el que estos departamentos funcionen como coordinadores de la actividad educativa desarrollada en el contexto geográfico del municipio y como programadores de una oferta de calidad. 


\section{Conclusión}

A la luz de los resultados aquí expuestos, podemos afirmar que la oferta en materia de educación a disposición de un ciudadano de un municipio rural con escasos recursos en nada se puede comparar con la extensa, rica y plural cantidad de programas, actividades y posibilidades a disposición de un ciudadano que habite una urbe (observamos diferencias de más de 65.000 actividades por año entre algunos departamentos). Y es esta desigualdad social manifiesta la que debería llevar a los responsables políticos a articular las medidas necesarias para garantizar la igualdad de oportunidades dentro de un Estado de derecho. Ante la inexistencia de una verdadera igualdad, niveladora del conjunto inicial de oportunidades entre ciudadanos, en ningún caso podremos considerarnos sujetos pertenecientes a un Estado verdaderamente justo, puesto que el principio básico de no vulnerabilidad parece no existir a la luz de los datos sobre Galicia recogidos en este estudio.

En definitiva, e intentando realizar un ejercicio de prospectiva en base a los datos y autores aquí citados, nos reafirmamos en que el futuro de estos departamentos en Galicia deberá enfocarse a la consecución de un trabajo colaborativo de cogestión de competencias entre la Consellería de Educación y unos DME de ámbito supramunicipal (comarcal, mancomunal, consorcios municipales...). Cogestión que, al igual que en otras comunidades autónomas españolas y regiones europeas, deberá caracterizarse por una segunda descentralización (entendida como coordinación y no simple delegación de competencias) y un considerable esfuerzo de trabajo horizontal (entre Administraciones locales, Autonómica, colectivos sociales,...). El objetivo es la consecución de Proyectos Educativos, a nuestro entender territoriales (supramunicipales), que posibiliten la optimización de los recursos existentes y la coordinación y corresponsabilidad de todos los agentes educativos de un territorio. Y es en este proceso donde los DME deberán jugar un papel clave.

\section{REFERENCIAS}

BOGDAN, R. C.; BIKLEN, S. K. Qualitative research for education: An introduction to theory and methods. Boston: Allyn and Bacon, Inc., 1982. 
BONAL, X. La equidad y la cohesión social en las políticas educativas: El papel del ámbito local. En: MERINO, A.; PLANA, J. (Coord.). La ciudad educa: Aportaciones para una política educativa local. Barcelona: Ediciones del Serbal, 2007.

CABALLO, M. B. (Coord.). O Eixo Atlántico: Un territorio educador, unha comunidade educativa. Vigo: Eixo Atlántico do Noroeste Peninsular, 2009.

CANDEDO, M. D.; MOSQUERA, M. G.; OJEA, R.; RODRÍGUEZ, X. M. Concellos e educación. En: CARIDE, J. A. (Dir.). A educación en Galicia. Problemas e perspectivas. Santiago de Compostela: USC, 1990.

COLINO, C.; DEL PINO, E. Democracia participativa en el nivel local: Debates y experiencias en Europa. Revista catalana de dret públic, v. 37, p. 247-283, 2008.

COSTA, A. Unha educación integrada no territorio. Perspectivas organizativo-administrativas. Materiais pedagóxicos, v. 1, p. 117-128, 1985.

DÍAZ-GIBSON, J.; CIVÍS, M.; CARRILLO, E.; CORTADA, M. El liderazgo y la gobernanza colaborativa en proyectos educativos comunitarios. Pedagogía Social. Revista Interuniversitaria, v. 26, p. 59-83, 2015.

ESPAÑA. Ley 7/1985, de 2 de abril, Reguladora de las Bases del Régimen Local. Boletín Oficial del Estado (España), p. 80, 3 abr. 1985.

ESPAÑA. Ley Orgánica 8/2013, de 9 de diciembre, para la Mejora de la Calidad Educativa. Boletín Oficial del Estado (España), p. 295, 10 dic. 2013a.

ESPAÑA. Ley 27/2013, de 27 de diciembre, de Racionalización y Sostenibilidad de la Administración Local. Boletín Oficial del Estado (España), p. 312, 30 dic. 2013b.

ESPEJO, B. (Coord.). Políticas educativas para el nuevo siglo. Salamanca: Espérides D. L., 2001.

GALAIS, C.; NAVARRO YÁÑEZ, C. J.; FONTCUBERTA, P. La calidad de los procesos participativos locales: Indicadores y factores explicativos contextuales. El caso de Andalucía. Revista Española de Ciencia Política, v. 32, p. 65-87, 2013.

GUBA, E. G.; LINCOLN, Y. S. Effetive evaluation: Improving the usefulness of evaluation resulta through responsive and naturalistic approaches. San Francisco: Jossey-Bass. (1. ed. 1981), 1992.

MÉNDEZ ROMEU, J. L. Políticas educativas de las ciudades. Eixo Atlántico, v. 2, p. 11-116, 2002.

MENDOZA, A. El estudio de casos. Un enfoque cognitivo. Alcalá de Guadaira (Sevilla): Trillas e MAD, 2006.

MONTERO, P.; CABALLO, M. B. La cooperación educativa en el territorio: Un estudio de la Eurorregión Galicia-Norte de Portugal. Cultura y Educación. Revista de teoría, investigación y practica, v. 23, n. 3, p. 431-443, 2011. 
MUÑOZ MORENO, J. L.; GAIRÍN SALLÁN, J. La implicación de los ayuntamientos en una educación descentralizada. Revista de Educación, v. 366, p. 165-188, 2014.

QUINTANA, T.; CASARES, M. La reforma del régimen local: Comentario a la Ley 27/2013, de 27 de diciembre, de racionalización y sostenibilidad de la Administración Local. Valencia: Tirant lo Blanch, 2014.

RODRÍGUEZ-ABELLA, X. M. Educación e cambio social: Achegas dos concellos nos procesos de produción de materiais curriculares. Santiago de Compostela: Tórculo. En: RODRÍGUEZ RODRÍGUEZ, X. (Coord.). Materiais curriculares e diversidade sociocultural. Santiago de Compostela: Tórculo, 2003.

SUBIRATS, J. Quina educació per quina societat. El paper dels Ajuntaments. DeProp. Revista de política educativa local. 2003. Disponible en: <www.deprop.net/cerca.asp? numrev $=26 \&$ lletra $=P \&$ cerca $=$ Participació $\% 20$ ciutadana $\&$ cnumrev $=6 \&$ sumari $=3 \&$ secc io $=0 \&$ numart $=5 \&$ versio $=1>$. Acceso en: 19 mayo 2016.

SUBIRATS, J. Once notas (dispersas) sobre el futuro de la educación. En: MERINO, A.; PLANA, J. (Coord.). La ciudad educa: Aportaciones para una política educativa local. Barcelona: Ediciones del Serbal, 2007.

VVAA. Varios Autores. I Xornadas galegas sobre concellos e educación. Santiago de Compostela: Minerva, 1985.

Texto recibido en 15 de junio de 2016. Texto aprobado en 16 de junio de 2016. 
